\section{Marta García Fernández}

Pontificia Universidad Comillas, Madrid mgafernandez@comillas.edu

ORCID: 0000-0002-8414-0996

DOI: http://dx.doi.org/10.12775/BPTH.2019.019
Biblica

et

Patristica

Thoruniensia

12 (2019) 4: 357-370

ISSN (print) $1689-5150$

ISSN (online) 2450-7059

\title{
La relectura del cuarto canto del Siervo en el judaísmo del Segundo Templo y en el NT
}

\author{
The Reading of the Servant's fourth Song \\ in the Second Temple's Judaism and in the NT
}

Resumen: La relectura del cuarto canto del Siervo en el judaísmo del Segundo Templo y en el NT ha sido una cuestión ampliamente estudiada y debatida. Por este motivo, partiendo del status quaestionis actual, el ensayo se circunscribe a ofrecer dos pistas que contribuyan a resituar el paradigma del Siervo isaiano como tipología cristológica de primera línea. Para ello en un primer apartado se afronta la espinosa cuestión que pone en relación al Mesías con el Siervo sufriente. En un segundo punto, teniendo en cuenta la íntima conexión entre el cuarto canto y el resto de poemas del Siervo, se insta a vislumbrar en los Evangelios una estructura análoga a la deuteroisaiana. Progresión que permite ahondar en una cristología del Siervo no limitada al aspecto sufriente.

\begin{abstract}
The reading of the Servant's fourth Song in the Second Temple's Judaism and in the NT has been a widely studied and debated issue. For this reason, based on the current status quaestionis, the essay is limited to offering two clues that contribute to place the paradigm of the Isaiah's Servant as a first line Christological typology. In a first section, we face the difficult question of the relation to the Messiah with the Suffering Servant. In a second moment, starting for the intimate conection between the fourth Song and the other Servant's poems, we'll glimpe an analogous Isaian structure in the Gospels. This progression allows to draw a Christology of the Servant not limited to the suffering aspect.
\end{abstract}

Palabras clave: Siervo sufriente; Mesías; Is 52,13-53,12; cantos del Siervo.

Keywords: Suffering Servant; Messiah; Isa 52,13-53,12; Servant’s Songs. 
T a cristología del Siervo de Yhwh, siendo una de las más trascendentales Lpara comprender la tipología veterotestamentaria sobre la que el NT dibuja y explica el acontecimiento Jesús de Nazaret, es en muchos casos relegada a un segundo plano frente a otros títulos considerados de primera línea tales como Mesías, Hijo del Hombre e Hijo de Dios. En correlación al llamamiento que realizó Walter Kasper acerca la centralidad que debía ocupar la misericordia en el Tratado de Dios ${ }^{1}$, a la exégesis actual se le podría instar a reconsiderar el lugar que le corresponde a este paradigma isaiano dentro de la teología neotestamentaria.

Este objetivo se halla como trasfondo del presente ensayo que, dada su limitada extensión, se cierne a apuntar dos aspectos que a nuestro juicio deberían ser revisados y con ello se ofrecen simultáneamente dos pistas hacia las que debería orientarse la investigación. El primero busca "desenmarañar" la enredada cuestión del Siervo sufriente equiparado al Mesías en el judaísmo del Segundo Templo. El segundo, no circunscribiendo la figura de este personaje al texto de Is $52,13-53,12$, apunta a la existencia de una estructura deuteroisaniana latente en los Evangelios.

\section{1. ¿Siervo vs Mesías?}

Es comúnmente aceptada la identificación de Jesús y el Siervo sufriente de Isaías, especialmente en lo que concierne a los relatos de la pasión y muerte. Sin embargo, si constatamos el número de citas referidas del cuarto Canto (Is 52,13-53,12) en las correspondientes secciones de los cuatro evangelios se reduce a una: Lc 22,37 (Is 53,12)2. El resto de comparecencias en los capítulos evangélicos de la pasión y muerte están catalogadas bajo el epígrafe de alusión. Entonces, ¿a qué se debe esta férrea correspondencia entre Jesús y el Siervo?

1 Cf. W. Kasper, La misericordia. Clave del Evangelio y de la vida cristiana, PT 193, Santander 2012, 15-19.

2 Según la edición crítica E. \& E. Nestle, B. \& K. Aland, J. Karavidopoulos, C. Martini, B.M. Metzger (ed.), Novum Testamentum Graece, Stuttgart ${ }^{27} 1993$ (NA ${ }^{27}$ ) y también para la edición de K. Aland, M. Black, C. Martini, B.M. Metzger, A. Wikgren (ed.), The Greek New Testament, Stuttgart ${ }^{4} 1993\left(\mathrm{GNT}^{4}\right)$ el número total de citas del cuarto canto del Siervo en los cuatro evangelios es de tres: Mt 8,17 (Is 53,4); Lc 22,37 (Is 53,12); In 12,38 (Is 53,1). De hecho, es notoria la escasez de referencias del cuarto canto en todo el NT en contraste con la repercusión que alcanzará en las disputas posteriores. Cf. P.N. DE Andrado, The Akedah Servant Complex. The Soteriological Linkage of Genesis 22 and Isaiah 53 in Ancient Jewish and Early Christian Writings, Leuven 2013, 24-27. 
Aunque la cita de Lc 22,37 está ubicada en un enclave estratégico particular y contiene una importancia inusitada ${ }^{3}$, probablemente el principal responsable de esta asociación haya que buscarlo en el episodio de Felipe y el etíope (Hch 8,26-40). Leyendo el pasaje de Is 53,7-8, este interroga al apóstol: «Te ruego de quién dice esto el profeta: ¿de sí mismo o de otro? Felipe entonces, partiendo del texto de la Escritura, se puso a anunciarle la Buena Nueva de Jesús» (Hch 8,34-35). En la obra lucana existe un cierto paralelismo entre este relato y el de Emaús. Allí también Jesús se hace el encontradizo en el camino e, igualmente, ante las dudas y desesperanzas de los discípulos, «empezando por Moisés y continuando por los profetas», les abre las Escrituras y les explica por qué «era necesario que el Mesías padeciera para entrar así en su gloria» (Lc 24,25-27).

\subsection{Hacia una comprensión más "elástica" de Mesías}

En ningún otro texto del NT es tan clara la identificación de Jesús con un personaje del AT como en Hch 8,34-35. Sin embargo, esta nitidez rápidamente se nubla al enredarse con la asociación, ya presente en Lc 24, que vincula dos figuras de por sí no conectadas: Mesías y Siervo sufriente ${ }^{4}$. Así pues, durante siglos la controversia entre el judaísmo y el cristianismo se cernió en legitimar o en deslegitimar el hecho de que el Mesías debía ser sufriente. Uno de los caminos transitados por la apologética judía fue evidenciar que las pruebas veterotestamentarias aducidas por los cristianos resultaban hermenéuticamente inadecuadas e insuficientes 5 . El otro camino recorrido, quizás más actual, es que la asociación entre Mesías y sufrimiento es preexistente al cristianismo ${ }^{6}$. Por tanto, el rechazo de Jesús como Mesías registrado en el NT no vendría motivado necesariamente por el hecho de haber muerto de manera ignominiosa.

3 Cf. P. Tremolada, "E fu annoverato fra iniqui". Prospettive di lettura della Passione second Luca alla luce di Lc 22,37 (Is 53,12d), AnBib 137, Roma 1997.

4 Cf. M. Wolf, R. Frydland, "Does Isaiah 53 speak of Jesus", American Messianic Jew 67.1 (1982) 13-15; A. Feuillet, "Une triple préparation du sacerdoce du Christ dans l'Ancient Testament (Melchisédech, Le Messie du Ps 110, le Serviteur d'Is 53", Divinitas 28.2 (1984) 103-136; R.A. Rosenberg, "The Slain Messiah in the Old Testament”, ZAW 99 (1987) 259-261.

5 Una muestra son las interesantes disputas exegéticas medievales representadas por la Escuela de Córdoba. Cf. F. Varo, Los cantos del siervo en la exégesis hispano-hebrea, Córdoba 1993.

6 Para una visión panorámica, cf. M. Henger, "The Effective History of Isaiah 53 in the Pre-Christian Period", en B. Janowski, P. Stuhlmacher (eds.), The Suffering Servant. Isaiah 53 in Jewish and Chrstian Sources, Grand Rapids 2004, 75-146. 
El problema es espinoso y antes de ofrecer soluciones, a mi juicio, es conveniente desenmarañarlo. En primer lugar, los estudios actuales muestran que la ardiente expectativa mesiánica de la que parece respirar el NT no fue compartida por todos los grupos judíos ${ }^{7}$. Es más, entre quienes esperaban una intervención de Dios en la historia no existía consenso sobre si actuaría directamente o a través de un mediador o Mesías ${ }^{8}$. En segundo lugar, entre aquellos que esperaban un Mesías existía disparidad de opiniones en referencia al número ${ }^{9}$, la identidad y misión del mismo ${ }^{10}$, el momento y modo de su venida ${ }^{11}$.

Aunque según Mc 8,27-33, los anuncios de pasión y muerte parecieran ser una especie de "correctivo" a una visión de mesianismo triunfalista compartida incluso por los discípulos, el amplio abanico de opciones pone de manifiesto que esta interpretación es una simplificación del vastísimo panorama religioso del s. I. Ahora bien, si entre todas las posibilidades existía la figura de un Mesías que, por implementar la restauración de Israel, iba a afrontar situaciones adversas y hostiles, cabe preguntarse cómo se llegó a formar esta idea.

7 "La centralización cristiana en la persona de Jesús ha llevado incorrectamente a considerar que el pensamiento judío veterotestamentario se centra en la persona del Mesías. Debe tenerse en cuenta que en el Antiguo Testamento el término ungido no se usa nunca para referirse a un salvador o redentor futuro, y que en los escritos judíos posteriores, del periodo comprendido entre el 200 a.C. y el 100 d.C. , sólo esporádicamente aparece el término en relación con agentes de salvación divina futura". Cf. L. Vegas, "La figura del 'Siervo de Yahweh" en A. Alonso (coord.), El mesianismo en el cristianismo antiguo y en el judaísmo, Valladolid 2000, 43-44.

8 Una visión panorámica se encuentra en A. Diez Macho, Apócrifos del Antiguo Testamento. Introducción general, vol. I., Madrid 1984, 351-389.

9 Así, por ejemplo, en los escritos de Qumrán detecta textos que mencionan una sola figura, dos o hasta tres Mesías. Cf. F. García Martínez, "Esperanzas mesiánicas en los escritos de Qumrán”, en F. García Martínez, J. Trebolle Barrera, Los hombres de Qumrán. Literatura, estructura social y concepciones religiosas, Madrid 1993, 187-222.

10 En correlación a la cuestión del número de Mesías, se halla su tipología bien de corte real, sacerdotal o profético. Es decir, las funciones que se le adscriben varían y van desde la restauración política a la religiosa. En F. García Martínez, "Esperanzas mesiánicas”, o.c., 187-188, n. 4, se ofrece una nota bibliográfica sobre los estudios más importantes sobre esta cuestión en Qumrán.

11 Cf. E. Puech, "Mesianismo, escatología y resurrección en los manuscritos del mar Muerto", en J. Trebolle Barrera (coord.), Paganos, judíos y cristianos en los textos de Qumrán, BCBO 5, Madrid 1999, 254-275. 


\subsection{El uso escatologizado de las Escrituras}

En el judaísmo del s. I las expectativas mesiánicas no eran compartidas por todos los grupos. Tampoco entre quienes la defendían el paradigma mesiánico mostraba contornos uniformes. Esta apertura tipológica era proclive a nuevas asociaciones sobre las que se discutía recurriendo a textos bíblicos, como se observa en Mc 12,35-37. A ello contribuyó una práctica hermenéutica presente en Qumrán. Los pesharim, o lecturas escatológicas judías de pasajes proféticos, dotaban de nuevos sentidos a perícopas que clásicamente no eran mesiánicas. Por ejemplo, las bendiciones de Jacob (Gn 49,10), el oráculo de Balaán $(\mathrm{Nm} 24,17)$, la profecía de Natán $(2 \mathrm{Sam} 7)$ y algunos salmos reales $(\mathrm{Sal} 2 ; 110)^{12}$.

En este contexto se podría encuadrar la identificación del Siervo de Yhwh con el Mesías. Ahora bien, sobre dicha asociación que aparece claramente en Lucas se discute su origen cristiano o pre-cristiano. La única prueba más directa de un antecedente judío es el Tárgum de Isaías: «He aquí mi siervo, el Mesías, prosperará, será exaltado, se acrecentará y será muy poderoso» (TgIs 52,13) ${ }^{13}$. Incluso con propuestas diferentes de datación, algunos estudiosos concuerdan que TgIs 52,13 es pre-cristiano o que, al menos, refleja una tradición previa a la polémica con el cristianismo. Si esto fuera así estaríamos ante una exégesis prematura que asocia la figura del Mesías con la del Siervo sufriente ${ }^{14}$. Pero no todos aceptan la datación temprana del Tárgum o que alguno de sus materiales recoja tradiciones tan antiguas.

No obstante, que no exista una relación directa de Is 52,13-53,12 con una interpretación mesiánica no excluye que pudiera existir la figura de un Mesías sufriente de corte profético precisamente como un desarrollo del pasaje de Dt 18,15.18 sobre el surgimiento de un profeta como Moisés y de Mal 3,23 acerca del retorno de Elías. Así en 1Q IX,11 aparece una especie de profeta escatológico ${ }^{15}$, como alternativa al mesianismo tradicional davídico (2Sam 7) y al sacerdotal (Jr 33,14-26; Zac 3; 6,9-14) ${ }^{16}$. Otros autores ven en la enigmática figura qumranita del Maestro de Justicia la tipología pre-cristiana del Mesías

12 Cf. F. García Martínez, “Esperanzas mesiánicas”, o.c., 189.

13 La traducción es de J. Ribera Florit, El Targum de Isaías. La versión aramea del profeta Isaías, Biblioteca Midrásica 6, Valencia 1988, 209.

14 Cf. B.D. Chilton, The Glory of Israel. The theology and provenience of the Isaiah Targum, JSOT.Sup. 23, Sheffield 1983, 90-92.

15 Cf. F. García Martínez, “Esperanzas mesiánicas”, o.c., 189.

16 Cf. L. Vegas, "La figura del Siervo”, o.c., 44-45. 
sufriente ${ }^{17}$. Pero la opinión generalizada es que las pruebas argüidas no son suficientes por el momento ni para negar ni para afirmar una concepción precristiana de Mesías sufriente ${ }^{18}$.

\subsection{El desarrollo de una teología del martirio}

En correlación a esta apertura escatológica de las Escrituras confluye el desarrollo de un nuevo planteamiento del sufrimiento. El exilio produce una primera reflexión sobre la crisis que supone que el justo padezca, tal como aparece registrado en libros como el de Jeremías, Job o algunos Salmos. Ahora bien, se observa un repunte de este aspecto en escritos tardíos ubicados al final de la época persa y en el convulso periodo heleno. Así Daniel (Dn 6-12), Ananías, Azarías y Misael (Dn 1-3), Susana (Dn 13), Mardoqueo en el libro de Ester, los hermanos Macabeos (2Mac 7) o el justo de Sabiduría (Sab 1,16-2,24; 3,1-9; 5,1-3) sufren por mantenerse fieles a su credo y convicciones. En correspondencia a este rebrote en la escritura canónica, se observa la misma tendencia en la literatura inter-testamentaria.

La difícil época de Antioco IV y la apostasía provocada por la violencia ejercida sobre Israel o por la fascinación hacia la cultura griega favoreció el desarrollo de una teología del martirio fruto de permanecer inamovibles en su fe (cf. Sab 3,1-9; $2 \mathrm{Mac} 7 ; 4 \mathrm{Mac}$ 17,20-22). La muerte leída en clave de sacrificio revierte en beneficio de otros. Por tanto, no se trata simplemente del esquema: vejación de un justo y posterior retribución, sino que estos "mártires" se constituyen en mediadores de salvación.

Teniendo en cuenta que el único texto del AT en el que aparece con claridad cómo este "intercambio" de mal revierte en bien para otros es el de Is 52,1353,12, es lógico pensar que la figura del Siervo de Yhwh hizo de potente catalizador sea de las figuras de justos anteriores como de inspiración del paradigma de justos posteriores. De hecho, a estas últimas se las dotó de un elemento inédito salvífico, ya que el cuarto canto puso las bases de la teología del martirio desde la categoría del sacrificio.

17 Así por ejemplo I. KNOHL, El mesías antes de Jesús. El siervo sufriente de los manuscritos del Mar Muerto, Biblioteca de ciencias bíblicas y orientales 8, Barcelona 2004. Carmignac considera que la tipología del Siervo estaba presente en Qumrán y ofrece un elenco de posibles alusiones. Cf. J. Carmignac, "Les citations de l'Ancient Testament et spécialment des poèmes de Serviteur dans les Hymnes de Qumran”, RQ 2 (1960) 383-393.

18 Cf. S.H.T. Page, “The Suffering Servant”, o.c., 481-497. 


\subsection{Releyendo y entrelazando personajes y textos}

Sin embargo, esta corriente no se conformará con elaborar una teología del justo que sufre y media la salvación sino que releerá desde esta clave martirial algunas historias patriarcales, sazonándolas con nuevos ingredientes que no gozan en el texto bíblico o potenciando elementos presentes en el pasaje, pero que pasan desapercibidos y que adquieren en este momento un mayor relieve. Así pues, en la literatura del Segundo Templo la figura de José será enriquecida con matices propios del siervo inocente que por envidia es tratado injustamente ${ }^{19}$. Óptica desde la cual, el NT lo acercará a Jesús ${ }^{20}$.

Es decir, la conexión de Jesús con la figura de José no adviene por una relación directa con el texto bíblico (Gn 37-50), sino desde el horizonte hermenéutico con que este patriarca es visto en el s. I dC.

Una reflexión análoga se podría hacer con Isaac en Gn 22. Dicho texto probablemente fue puesto en conexión con Is $52,13-53,12^{21}$, dando lugar a una potente tradición conocida con el nombre de Aqedáh ${ }^{22}$. La característica más notoria de esta teología es el papel activo que juega Isaac en el sacrificio. Este hecho sumado a la vinculación del patriarca con el cordero que se inmolará en sustitución de su persona, pone en relación a Gn 22 con la fiesta de la Pascua (Ex 12,1-14) y con Dt 15,19-20, ya que los primogénitos pertenecen al Señor. Por eso, algunos estudiosos consideran que Gn 22 es una narración etiológica que pretende justificar la sustitución del sacrificio humano por el cordero ${ }^{23}$. La estrecha relación entre la soteriología de la muerte de Jesús y la Aqedáh, junto a la datación de los textos de los que hasta el momento se disponían, llevó a pensar que se trataba de una tradición judía post-cristiana. Sin embargo, el descubrimiento del manuscrito 4Q225 ha modificado esta visión ${ }^{24}$.

Concluyendo, aunque los datos no permiten dirimir la cuestión sobre si la asociación del Mesías con Is 52,13-53,12 fue cristiana o pre-cristiana, dicha

19 Cf. M.J. Guevara Llaguno, Esplendor en la diáspora. La historia de José (Gn 37-50) y sus relecturas en la literatura bíblica y parabíblica (BM 29), Estella 2006.

20 Cf. I. Angulo Ordorika, “No habéis leído estas Escritura?” (Mc 12,10). El trasfondo veterotestamentario como clave hermenéutica de Mc 12,1-12, Disertación defendida en la Pontificia Universidad Comillas, Madrid 2018, 322-333 (en fase de publicación).

21 Sobre esta cuestión existe una interesante monografía, cf. P.N. DE Andrado, The Akedah Servant Complex, o.c.

22 Cf. J. Swetnam, Jesus and Isaac. A Study of the Epistle to the Hebrew in the Light of the Aquedah, AnBib 94, Rome 1981; I. Kalimi, “"'Go, I Beg You. Take Your Beloved Son and Stay Him!". The Binding of Isaac in Rabbinic Literature and Thought", RRJ 13 (2010) 1-29.

23 Cf. I. Angulo Ordorika, “'No habéis leído estas Escritura?” (Mc 12,10), o.c., 313-322.

24 Cf. P.N. DE Andrado, The Akedah, o.c., 108-115. 
conexión es favorecida por un ambiente en el que la tipología mesiánica no es unánime y, además, existe una apertura escatológica de los textos que amplía con nuevos pasajes y personajes el catálogo mesiánico. Es más, la teología del martirio potencia el efecto y provoca una nueva relectura de figuras como José e Isaac desde esta clave hermenéutica. Se entrelazan así personajes como Isaac y el Siervo de por sí conectados, y se construye una tipología de mediador "mártir" que es plausible que se asociara al Mesías, dada su intervención soteriológica crucial en la historia. En consecuencia, para un correcto estudio de la presencia del cuarto canto del Siervo en el NT habría que tener en cuenta el humus hermenéutico en el que se fraguan los textos, así como la amplia constelación asociativa de tipologías y pasajes que se entrelazan.

\section{Un esquema compartido y latente}

La asociación entre el Siervo y Jesús no se debería agotar en la cuestión del siervo sufriente, ya que Is 52,13-53,12 es el último de cuatro cantos que, en opinión de muchos se hayan conectados no solo entre ellos ${ }^{25}$ sino con su contexto ${ }^{26}$. Esto es, la relación de Cristo con el ebed ’ădōnāy se ha circunscrito al ámbito de la pasión y muerte, difuminando la profunda conexión entre estas y una vida entendida como servicio, puesto que los evangelios entablan una sinonimia entre servir y dar la vida (Mc 10,45), entre curar y cargar con el dolor (Mt 8,17), entre lavar los pies y la Eucaristía (Jn 13). Por eso, como retiene Martin Hengel habría que ir más allá del simple pasaje y considerar el texto de Isaías como una unidad, tal como se entendía en la antigüedad ${ }^{27}$.

\subsection{Una progresión implícita en Deutero-Isaías}

El Segundo Isaías, sección en la que se encuentra los cuatro cantos del siervo (Is 42,1-4; 49,1-6; 50,4-9; 52,13-53,12), comienza con un imperativo, consolad a mi pueblo (Is 40,1), que introduce una tensión dramática, ya que la consola$36-58$.

25 Cf. O.H. Steck, “Aspekte des Gottesknechtes in Jes 52,13-53,12”, ZAW 97 (1985)

26 Cf. C.R. North, The Suffering Servant in Deutero-Isaiah. An Historical and Critical Study, Oxford ${ }^{2} 1963$, 161-178; T.N.D. Mettinger, A Farewell to the Servant Songs. A Critical Examination of an Exegetical Axiom, Scripta Minora, Lund 1983.

27 Cf. M. Henger, “The Effective History of Isaiah 53”, o.c., 79. 
ción no llega hasta Is $54-55^{28}$. Por este motivo, algunos autores consideran Is 52,13-53,12 la puerta de acceso a la Nueva Alianza que se opera misteriosamente por este mediador que es el ebed '̆a $\underline{d} \bar{o} n \bar{a} y^{29}$. Sin embargo, uno de los aspectos más llamativos de esta parte de Isaías es la resistencia que ofrece Israel para acoger el mensaje del profeta. Pues no se trata de una denuncia sino de un anuncio de salvación que, además, pide ser recibido en una situación dolorosa como fue el exilio.

De hecho, entre la rica argumentación que Yhwh despliega para convencer a un Israel ofendido con la forma de actuar de Dios (Is 40,27; 45,9-13), se integra la figura de un siervo quien lleva adelante el querer de Dios y su mensaje. Pues bien, al igual que sucede en las confesiones de Jeremías ${ }^{30}$, se podría decir que también entre los cuatro cantos del Siervo existe una progresión que consiste en un incremento de rechazo y tensión hacia su persona. Es decir, en la medida que se incide en la oferta de perdón, se observa que va creciendo la violencia hacia esta figura.

Así pues en el primer canto prácticamente no hay vestigios negativos, salvo interpretar que la caña cascada que no se quiebra y el pábilo vacilante que no se apaga (Is 42,3) es el Siervo ${ }^{31}$. En el segundo canto se observa un vocabulario que apunta cierta hostilidad: "hizo de mi boca una espadas afiliada, me escondió en la sombra de su mano» (Is 49,2), más las palabras adscritas en estilo directo que muestran cierta desesperanza del 'ebed: «en vano me he cansado, en viento y nada he gastado mis fuerzas» (Is 49,3). En el tercer canto a partir del v.6 comienza los ultrajes hacia siervo (Is 50,6-9). Hostilidad y violencia que se consuman en Is 52,13-53,12.

Este último poema, sin embargo, es formulado como una confesión. Es decir, la comunidad que habla lo hace para indicar que se confundieron y que, pensando que era un hombre despreciado por Dios, en cambio, en él Yhwh estaba salvándoles. De ahí, que el texto se muestra interesado en que otros también se adhieran a esta confesión (Is 53,1), dando así la sensación de

28 Cf. H.-J. Hermisson, "Einheit und Komplexität Deuterojesajas. Probleme der Redaktionsgeschichte von Jes 40-55", en J. Vermeylen, ed., The Book of Isaiah. Le Livre d'Isaïe. Les oracles et leurs relectures. Unité et complexité de l'ouvrage, BEThL 81, Leuven 1989,290 .

29 Cf. R.F. Melugin, The Formation of Isaiah 40-55, BZAW 141, Berlin 1976, 174; M. Garcia Fernandez, Consolad, consolad a mi pueblo. El tema de la consolación en Deuteroisaías, AnBib 181, Roma 2010, 236.

30 Cf. J.L. Sicre, Profetismo en Israel. El Profeta. Los Profetas. El mensaje, Estella 1992, $130-135$.

31 Cf. H. Simian-Yofre, Sofferenza dell'uomo e silenzio di Dio nell'Antico Testamento e nella letteratura del Vicino Oriente Antico, Studia Biblica 2, Roma 2005, 152. 
que la acción salvífica del Siervo se prolongará en el tiempo ${ }^{32}$. Es más, ubicado precisamente antes de los capítulos de Nueva Alianza, donde se despliega la consolación prometida (Is 54-55), el pasaje constituye la puerta de acceso a la misma. Esto es, Israel viendo morir a este hombre deformado sale de la dureza del sufrimiento que les ha provocado el exilio y puede reconciliarse con Dios ${ }^{33}$.

\section{2. ¿Una progresión implícita en los Evangelios?}

El esquema sinóptico latente del ministerio de Jesús guarda una cierta similitud con el eje que atraviesa DtIs. Pues su misión se presenta como una oferta de salvación que paradójicamente, no solo no es acogida sino que además produce un proceso de endurecimiento cada vez mayor por parte de las autoridades que culmina con su ejecución. Precisamente, en esta forma de morir se revela el sentido profundo de toda la existencia de Jesús entregada al servicio de los demás.

Pues bien, a pesar de la problemática inherente a las citas y de que el confín entre cita y alusión es un terreno discutido ${ }^{34}$, si nos atenemos a la propuesta de $\mathrm{NA}^{27}$ observamos que en los sinópticos subyace una estructura básica que recuerda a la progresión de los cantos del Siervo referidos en DtIs. De hecho, salvo raras excepciones, las citas o alusiones a Is 50,4-11 e Is 52,13-53,12 se concentran en los capítulos de pasión y muerte, mientras que en el momento del Bautismo (Mt 3,17; Mc 1,11; Lc 3,22) se halla una alusión a Is 42,1. Al entrar en la vida pública Jesús es introducido por el Padre a través de una revelación de su identidad y misión. Aunque en esta carta de presentación se combine Is 42,1 con el Sal 2,7 y Gn 22, este vestigio del primer canto indicaría que Jesús es comprendido desde la tipología del Siervo de Yhwh algo que se refuerza con su muerte.

Marcos es el evangelio que cuenta con menos alusiones a los cantos del Siervo, pero es quizás el que mantiene una estructura más clara. Existe una inclusión entre la afirmación del Padre en Bautismo (Mc 1,11) y la del centurión (Mc 15,38). El hecho de esta confesión sea formulada inmediatamente después de la muerte, evoca al cuarto canto, ya que allí la comunidad confiesa

32 M. García Fernández, Consolad, o.c. 254-255.271.

33 En cierto modo, 2Cor 5,18 recurre a una dinámica parecida. Cf. J.M. Granados, La reconciliación en la Carta a los Efesios y en la Carta a los Colosenses, AnBib 170, Roma 2008, 87.

34 Cf. B.D. Sommer, "Allusions and Illusions. The Unity of the Book of Isaiah in Light of Deutero-Isaiah's Use of Prophetic Tradition”, en R.F. Melugin, M.A. Sweeney, eds., New Visions of Isaiah, JSOT.S 214, Sheffield 1996, 156-186. 
su equivocación precisamente tras su óbito (Is 53,4-5). En cierto modo, como apunta 2Cor 5,18-20, es como si verle morir les sacara de la dureza en la que se encontraban y pudieran admitir el sentido oblativo de su vida.

Salvo la cita explícita de Lc 22,37, los sinópticos aluden al cuarto canto en episodios paralelos a lo largo de los relatos de la pasión. Sin embargo, en relación a la declaración del Bautismo, Mateo y Lucas amplían el radio: sea en referencia a su identidad y misión, que en los textos de infancia lucana se sintetiza en ser "luz de las naciones" (Lc 1,79; 2,30.32; cf. Is 42,6 e Is 49,6); sea en relación a un mensaje que se equipara al de Is 61 , especialmente en lo que concierne a las bienaventuranzas (Mt 5,3-4; Lc 6,20-21), pero también porque el cumplimiento de esta Escritura acredita ante el Bautista que Él es quien tenía que venir (Mt 11,5; Lc 7,22).

Además de estas referencias, en el corpus de cada sinóptico la $\mathrm{NA}^{27}$ descubre alusiones al tercer o cuarto canto del siervo que, a nuestro juicio, contribuyen a una profundización cristológica desde la tipología del 'ebed de Yhwh. Así pues, en el corpus marcano hallamos dos nuevas alusiones al tercer (Is 50,6) y cuarto canto (Is 53,3) precisamente en o entre los anuncios de la pasión (Mc 9,12; 10,34). Especialmente es significativo el último, puesto que equipara el servir con entregar la vida (Mc 10,45//Is 53,11). Es decir, el servicio de Jesús no es un acto puntual está conectado con su final. El sentido de su existencia es entregarla por otros.

Mateo, en cambio, une la cristología del siervo al tema de las curaciones, dándoles una mayor hondura. De hecho, tras la polémica curación en sábado se cita a Is 42,1-3 (Mt 12,18-20). Y con ello se indica que, al igual que el programa del 'ebed de Isaías, su forma de instaurar el Reino tampoco será violenta, pues no quebrará la caña cascada. Es más, para culminar el primer ciclo de tres milagros se cita Is 53,4 (Mt 8,17). De esta manera, se pone en conexión las curaciones con el misterio de la pasión. En definitiva Jesús cura porque carga con el pecado y muere por nosotros.

La obra lucana es la que explícitamente equipara al siervo con Jesús. El texto más emblemático es el de Hch 8,34-35. Pero también el episodio de la sinagoga de Nazaret (Lc 4,16-25), en el que se indica que la lectura de Is 61,1-2 se cumple en Él, ocupa un lugar programático, por la importancia del momento inaugural pero también porque hace inclusión con el pasaje de Emaús (Lc 24,13-35). De hecho, tanto allí como en el camino de Emaus Jesús abre la Escritura y se presenta como la clave hermenéutica de la misma. En su persona se inaugura el año de gracia (Lc 4), pero para ello será necesario que el Mesías padezca (Lc 24). Esta conexión se refuerza ulteriormente en la relación presente entre la confesión de su identidad en el Bautismo (Lc 3,22) y la Transfiguración (Lc 9,35). Es más, justo cuando se inicia el camino hacia Jerusalén 
(Lc 9,51), el texto indica que Jesús "endurece el rostro", evocando con esta expresión a Is 50,6 $6^{35}$. Es decir, Jesús se prepara para afrontar la Pasión como el siervo de Yhwh.

El evangelio de Juan se distancia de los sinópticos relativamente. Pues la alusión programática al "cordero de Dios" (Jn 1,29), en opinión de $\mathrm{NA}^{27}$ evoca Is 53,6-7. Posiblemente Juan conecta aquí con la tradición de la Aqedáh, ya que la palabra aramea "cordero" (טלה) y "siervo" (טלי) son homófonas y se asocian a la Pascua. De ahí, que Jn 1,29 haga inclusión con la pasión. También en otro lugar programático se evoca la muerte de Jesús desde la óptica de la glorificación: el Hijo del Hombre será levantado (Jn 3,14). Este verbo alude la promesa de exaltación de Is 52,13 . Es más, como en los sinópticos, también en Juan existe un triple anuncio pero de glorificación (Jn 3,14; 8,28; 12,32-34), perspectiva desde la cual se presenta la muerte de Jesús. Por último, se halla una cita explícita a Is 53,1 justo antes de entrar en la pasión y para cerrar el libro de los signos. Se trata de una pregunta: «¿quién creyó a nuestra predicación?», que pone en el candelero el drama de la dureza de Israel para aceptar a un Mesías sufriente (Jn 12,34).

En conclusión, podríamos decir que sería necesario realizar un estudio más exhaustivo de las citas y alusiones de los cantos del siervo a lo largo de los Evangelios, así como de la maraña tipológica con la que esta figura se entrelaza. No obstante, la batida realizada nos ofrece una primera panorámica para concluir que la relación entre Jesús y el Siervo no se circunscribe al ámbito de la pasión y muerte. Es más, existe una progresión análoga a la de Isaías que invita a repensar una cristología del Siervo.

\section{Bibliografía}

Aland K., Black M., Martini C. , Metzger B.M., Wikgren A. (ed.), The Greek New Testament, Stuttgart ${ }^{4} 1993\left(\mathrm{GNT}^{4}\right)$.

Angulo Ordorika I., “No habéis leído estas Escritura?” (Mc 12,10). El trasfondo veterotestamentario como clave hermenéutica de Mc 12,1-12, Disertación defendida en la Pontificia Universidad Comillas, Madrid 2018, 322-333.

Carmignac J., "Les citations de l'Ancient Testament et spécialment des poèmes de Serviteur dans les Hymnes de Qumran", RQ 2 (1960) 383-393.

Chilton B.D., The Glory of Israel. The theology and provenience of the Isaiah Targum, JSOT.Sup. 23, Sheffield 1983.

De Andrado P.N., The Akedah Servant Complex. The Soteriological Linkage of Genesis 22 and Isaiah 53 in Ancient Jewish and Early Christian Writings, Leuven 2013.

35 Cf. P. Tremolada, "E Fu annoverato fra iniqui”, o.c., 142. 
Diez Macho A., Apócrifos del Antiguo Testamento. Introducción general, vol. I., Madrid 1984.

Feuillet A., "Une triple préparation du sacerdoce du Christ dans l'Ancient Testament (Melchisédech, Le Messie du Ps 110, le Serviteur d'Is 53", Divinitas 28.2 (1984) 103-136.

Garcia Fernandez M., Consolad, consolad a mi pueblo. El tema de la consolación en Deuteroisaías, AnBib 181, Roma 2010.

García Martínez F., "Esperanzas mesiánicas en los escritos de Qumrán”, en F. García Martínez, J. Trebolle Barrera, Los hombres de Qumrán. Literatura, estructura social y concepciones religiosas, Madrid 1993, 187-222.

Granados J.M., La reconciliación en la Carta a los Efesios y en la Carta a los Colosenses, AnBib 170, Roma 2008.

Guevara Llaguno M.J., Esplendor en la diáspora. La historia de José (Gn 37-50) y sus relecturas en la literatura bíblica y parabíblica (BM 29), Estella 2006.

Henger M., “The Effective History of Isaiah 53 in the Pre-Christian Period”, en B. Janowski, P. Stuhlmacher (eds.), The Suffering Servant. Isaiah 53 in Jewish and Chrstian Sources, Grand Rapids 2004, 75-146.

Hermisson H.-J., "Einheit und Komplexität Deuterojesajas. Probleme der Redaktionsgeschichte von Jes 40-55”, en J. Vermeylen, ed., The Book of Isaiah. Le Livre d'Isaïe. Les oracles et leurs relectures. Unité et complexité de l'ouvrage, BEThL 81, Leuven 1989.

Kalimi I., “'Go, I Beg You. Take Your Beloved Son and Stay Him!'. The Binding of Isaac in Rabbinic Literature and Thought", RRJ 13 (2010) 1-29.

Kasper W., La misericordia. Clave del Evangelio y de la vida cristiana, PT 193, Santander 2012.

Knohl I., El mesías antes de Jesús. El siervo sufriente de los manuscritos del Mar Muerto, Biblioteca de ciencias bíblicas y orientales 8, Barcelona 2004.

Melugin R.F., The Formation of Isaiah 40-55, BZAW 141, Berlin 1976.

Mettinger T.N.D., A Farewell to the Servant Songs. A Critical Examination of an Exegetical Axiom, Scripta Minora, Lund 1983.

Nestle E. \& E., Aland B. \& K., Karavidopoulos J., Martini C. , Metzger B.M. (ed.), Novum Testamentum Graece, Stuttgart ${ }^{27} 1993\left(\mathrm{NA}^{27}\right)$.

North C. R., The Suffering Servant in Deutero-Isaiah. An Historical and Critical Study, Oxford ${ }^{2} 1963$.

Puech E., "Mesianismo, escatología y resurrección en los manuscritos del mar Muerto", en J. Trebolle Barrera (coord.), Paganos, judíos y cristianos en los textos de Qumrán, BCBO 5, Madrid 1999, 254-275.

Ribera Florit J., El Targum de Isaías. La versión aramea del profeta Isaías, Biblioteca Midrásica 6, Valencia 1988.

Rosenberg R.A., “The Slain Messiah in the Old Testament”, ZAW 99 (1987).

Sicre J.L., Profetismo en Israel. El Profeta. Los Profetas. El mensaje, Estella 1992.

Simian-Yofre H., Sofferenza dell'uomo e silenzio di Dio nell'Antico Testamento e nella letteratura del Vicino Oriente Antico, Studia Biblica 2, Roma 2005. 
Sommer B.D., "Allusions and Illusions. The Unity of the Book of Isaiah in Light of Deutero-Isaiah's Use of Prophetic Tradition", en R.F. Melugin, M.A. Sweeney, eds., New Visions of Isaiah, JSOT.S 214, Sheffield 1996, 156-186.

Steck O.H., "Aspekte des Gottesknechtes in Jes 52,13-53,12", ZAW 97 (1985) 36-58.

Swetnam J., Jesus and Isaac. A Study of the Epistle to the Hebrew in the Light of the Aquedah, AnBib 94, Rome 1981.

Tremolada P., "E fu annoverato fra iniqui". Prospettive di lettura della Passione second Luca alla luce di Lc 22,37 (Is 53,12d), AnBib 137, Roma 1997.

Varo F., Los cantos del siervo en la exégesis hispano-hebrea, Córdoba 1993.

Vegas L., "La figura del 'Siervo de Yahweh", en A. Alonso (coord.), El mesianismo en el cristianismo antiguo y en el judaísmo, Valladolid 2000.

Wolf M., Frydland R., "Does Isaiah 53 speak of Jesus", American Messianic Jew 67.1 (1982). 